\title{
Characteristic Analysis of White Gaussian Noise in S-Transformation Domain
}

\author{
Xinliang Zhang ${ }^{1}$, Yue $\mathbf{Q i}^{1}$, Mingzhe Zhu ${ }^{1,2}$ \\ ${ }^{1}$ Department of Electronic Engineering, Xidian University, Xi'an, China; ${ }^{2}$ The Ministry Key Laboratory of Electronic Information \\ Countermeasure and Simulation, Xidian University, Xi'an, China. \\ Email: zxl_xddc@foxmail.com
}

Received October 2013

\section{ABSTRACT}

The characteristic property of white Gaussian noise (WGN) is derived in S-transformation domain. The results show that the distribution of normalized S-spectrum of WGN follows $\chi^{2}$ distribution with two degrees of freedom. The conclusion has been confirmed through both theoretical derivations and numerical simulations. Combined with different criteria, an effective signal detection in S-transformation can be realized.

\section{KEYWORDS}

\section{Signal Detection; S-Transform; White Gaussian Noise; $\chi^{2}$ Distribution}

\section{Introduction}

THE S-transform(ST) has been well studied since it was proposed by R. G. Stockwell in 1996 [1] and has already found applications in many fields such as geophysics [2], mechanical systems [3] and medical signal analyses [4]. The signal processing framework in S-transformation domain is under building and more and more ST based methods are proposed. The most contributive works among them were conducted by C. R. Pinnegar who extended the original ST to generalized ST [2] and presented several kinds of useful STs for specific conditions, such as the asymmetric ST [2], the bi-Gaussian ST [5] and the complex ST [6]. Other outstanding works were concentrated on ST based time-frequency filtering [7], the side effects of inverse ST [8], ST based instantaneous frequency estimation [9] and ST based pattern recognition [10,11]. However, the noise analysis in S-transformation domain, which is the essential problem for signal detection, has not been well studied yet. [11] presented an illuminating idea for noise distribution in a specific generalized ST domain but didn't prove it theoretically. In this paper, the characteristic property of WGN in original ST domain is derived theoretically and the conclusion is further verified by Monte Carlo method. Its application in nonstationary signal detection is also illustrated.

The rest of this paper is organized into as follows: In
Section 2, the proposition of WGN distribution followed by its theoretical derivation is given. Section 3 verifies the proposition by Monte Carlo method. In Section 4, the performance of signal detection is illustrated by detecting transient sinusoid signals under the constant-false-alarmrate (CFAR) criterion. Finally, conclusions are drawn in Section 5 .

\section{Characteristic Property of WGN in ST Domain}

\subsection{Proposition}

The S-transform of a signal $x(t)$ is defined by [1]

$$
S_{x}(t, f)=\int_{-\infty}^{\infty} x(\tau) \frac{1}{\sqrt{2 \pi}} \exp (-j 2 \pi f \tau) w(t-\tau, f) d \tau
$$

where the window function is

$$
w(\tau, f)=|f| \exp \left(-\tau^{2} f^{2} / 2\right)
$$

And the characteristic and the advantage of ST has been sufficiently described by previous literature [2-6].

Let $\left|S_{x}(t, f)\right|^{2}$ be the S-spectrum of $x(t)$ and white Gaussian noise $n(t) \sim N\left(0, \sigma^{2}\right)$; then the normalized S-spectrum of $n(t), 2\left|S_{n}(t, f)\right|^{2} / E\left[\left|S_{n}(t, f)\right|^{2}\right]$ (denoted as $\left|N S_{n}(t, f)\right|^{2}$ ), follows $\chi^{2}$ distribution with two degree of freedom. 


\subsection{Proof}

Step.1: Derive the distribution of $\operatorname{Re}\left[S_{n}(t, f)\right]$ and $\operatorname{Im}\left[S_{n}(t, f)\right]$ and prove that they follow the identical Gaussian distribution.

By definition, the S-spectrum of $n(t)$ can be expressed as

$$
\left|S_{n}(t, f)\right|^{2}=\operatorname{Re}^{2}\left[S_{n}(t, f)\right]+\operatorname{Im}^{2}\left[S_{n}(t, f)\right]
$$

Before further proceeding, let us analyze the distribution of $\operatorname{Re}\left[S_{n}(t, f)\right]$ and $\operatorname{Im}\left[S_{n}(t, f)\right]$, respectively. The real part of $S_{n}(t, f)$ is given by

$\operatorname{Re}\left[S_{n}(t, f)\right]=\int_{-\infty}^{\infty} \frac{|f|}{\sqrt{2 \pi}} n(\tau) \exp \left(\frac{-(t-\tau)^{2} f^{2}}{2}\right) \cos (2 \pi f \tau) d \tau$

$\operatorname{Re}\left[S_{n}(t, f)\right]$ is the integral of $n(t)$ multiplied by deterministic signals, thus the distribution of $\operatorname{Re}\left[S_{n}(t, f)\right]$ is the same as $n(t)$ with zero mean and its variance $\sigma_{R}^{2}(t)$ expressed as

$$
\begin{aligned}
\sigma_{R}^{2}(t)= & E\left[\int_{-\infty}^{\infty} \frac{|f|}{\sqrt{2 \pi}} n\left(\tau_{1}\right) \exp \left(\frac{-\left(t-\tau_{1}\right)^{2} f^{2}}{2}\right) \cos \left(2 \pi f \tau_{1}\right) d \tau_{1}\right. \\
& \left.\cdot \int_{-\infty}^{\infty} \frac{|f|}{\sqrt{2 \pi}} n^{*}\left(\tau_{2}\right) \exp \left(\frac{-\left(t-\tau_{2}\right)^{2} f^{2}}{2}\right) \cos \left(2 \pi f \tau_{2}\right) d \tau_{2}\right] \\
= & \left(\frac{|f|}{\sqrt{2 \pi}}\right)^{2} \int_{-\infty}^{\infty} \int_{-\infty}^{\infty} E\left[n\left(\tau_{1}\right) n^{*}\left(\tau_{2}\right)\right] \cdot \exp \left(\frac{-\left(t-\tau_{1}\right)^{2} f^{2}}{2}\right) \\
& \cdot \exp \left(\frac{-\left(t-\tau_{2}\right)^{2} f^{2}}{2}\right) \cdot \cos \left(2 \pi f \tau_{1}\right) \cdot \cos \left(2 \pi f \tau_{2}\right) d \tau_{1} d \tau_{2} \\
= & \sigma^{2}\left(\frac{|f|}{\sqrt{2 \pi}}\right)^{2} \int_{-\infty}^{\infty} \exp \left(-(t-\tau)^{2} f^{2}\right) \cos { }^{2}(2 \pi f \tau) d \tau \\
= & \sigma^{2}\left(\frac{|f|}{\sqrt{2 \pi}}\right)^{2} \int_{-\infty}^{\infty} \exp \left(-(t-\tau)^{2} f^{2}\right) \cdot\left(\frac{\cos (4 \pi f \tau)+1}{2}\right) d \tau \\
= & \frac{1}{2} \sigma^{2} \cdot\left(\frac{|f|}{\sqrt{2 \pi}}\right)^{2}\left(\int_{-\infty}^{\infty} \exp \left(-(t-\tau)^{2} f^{2}\right) \cdot \cos (4 \pi f \tau) d \tau\right. \\
& \left.+\int_{-\infty}^{\infty} \exp \left(-(t-\tau)^{2} f^{2}\right) d \tau\right) \\
= & \frac{1}{2} \sigma^{2} \cdot \frac{|f|}{2 \sqrt{\pi}}\left(\exp \left(-4 \pi^{2}\right) \cos (4 \pi f t)+1\right)
\end{aligned}
$$

Similarly, the imaginary part $\operatorname{Im}\left[S_{n}(t, f)\right]$ follows the Gaussian distribution with $\operatorname{Im}\left[S_{n}(t, f)\right] \sim N\left(0, \sigma_{I}^{2}(t)\right)$, where

$$
\sigma_{I}^{2}(t)=\frac{1}{2} \sigma^{2} \cdot \frac{|f|}{2 \sqrt{\pi}}\left(1-\exp \left(-4 \pi^{2}\right) \cos (4 \pi f t)\right)
$$

In Equation (5) and Equation (6), the constant term, $\exp \left(-4 \pi^{2}\right) \approx 7.1572 \mathrm{e}-18$, is of very small value which can approximately equal to zero, thus $S_{n}(t, f)$ can be regarded as having identically distributed real and imaginary parts, that is, $\operatorname{Re}\left[S_{n}(t, f)\right]$ and $\operatorname{Im}\left[S_{n}(t, f)\right]$ $\sim N\left(0, \frac{1}{2} \sigma^{2} \cdot \frac{|f|}{2 \sqrt{\pi}}\right)$.

Step.2: Derive the expression of the average S-spectrum of $n(t), E\left[\left|S_{n}(t, f)\right|^{2}\right]$, and Prove that

$\operatorname{Re}\left[N S_{n}(t, f)\right]$ and $\operatorname{Im}\left[N S_{n}(t, f)\right]$ follow identical standard normal distribution.

The normalized S-spectrum of $n(t)$ can be written as

$$
\begin{aligned}
\left|N S_{n}(t, f)\right|^{2} & =2 \cdot\left(\frac{\operatorname{Re}^{2}\left[\left|S_{n}(t, f)\right|\right]}{E\left[\left|S_{n}(t, f)\right|^{2}\right]}+\frac{\operatorname{Im}^{2}\left[\left|S_{n}(t, f)\right|\right]}{E\left[\left|S_{n}(t, f)\right|^{2}\right]}\right) \\
& =\operatorname{Re}^{2}\left[N S_{n}(t, f)\right]+\operatorname{Im}^{2}\left[N S_{n}(t, f)\right]
\end{aligned}
$$

Since the average S-spectrum of $n(t)$,

$$
\begin{aligned}
E\left[\left|S_{n}(t, f)\right|^{2}\right], & \text { is given by } \\
E\left[\left|S_{n}(t, f)\right|^{2}\right] & =\iint_{\infty} E\left[n\left(\tau_{1}\right) n^{*}\left(\tau_{2}\right)\right] w\left(\tau_{1}-t, f\right) \\
& w\left(\tau_{2}-t, f\right) \exp \left(-2 \pi f j\left(\tau_{1}-\tau_{2}\right)\right) d \tau_{1} d \tau_{2}( \\
& =\frac{|f|}{2 \sqrt{\pi}} \sigma^{2}
\end{aligned}
$$

then the distribution of $\operatorname{Re}\left[N S_{n}(t, f)\right]$ and $\operatorname{Im}\left[N S_{n}(t, f)\right]$ can be obtained as

$$
\operatorname{Re}\left[N S_{n}(t, f)\right] \sim N(0,1) ; \operatorname{Im}\left[N S_{n}(t, f)\right] \sim N(0,1)
$$

Step.3: Prove that $\operatorname{Re}\left[N S_{n}(t, f)\right]$ and $\operatorname{Im}\left[N S_{n}(t, f)\right]$ are independent.

To determine the distribution of $\left|N S_{n}(t, f)\right|^{2}$, the correlation of $\operatorname{Re}\left[N S_{n}(t, f)\right]$ and $\operatorname{Im}\left[N S_{n}(t, f)\right]$ need to be analyzed. The correlation function is

$$
\begin{aligned}
R_{R I}\left(t_{1}, t_{2}\right)= & E\left\{\operatorname{Re}\left[N S_{n}\left(t_{1}, f\right)\right] \cdot \operatorname{Im}\left[N S_{n}\left(t_{2}, f\right)\right]\right\} \\
= & 2 \cdot \frac{|f|}{\sigma^{2} \sqrt{\pi}} \int_{-\infty}^{\infty} \int_{-\infty}^{\infty} E\left[n\left(\tau_{1}\right) n^{*}\left(\tau_{2}\right)\right] \exp \left(\frac{-f^{2}\left(t_{1}-\tau_{1}\right)^{2}}{2}\right) \\
& \cdot \exp \left(\frac{-f^{2}\left(t_{2}-\tau_{2}\right)^{2}}{2}\right) \cdot \cos \left(2 \pi f \tau_{1}\right) \cdot \sin \left(2 \pi f \tau_{2}\right) d \tau_{1} d \tau_{2} \\
= & 2 \cdot \frac{|f|}{\sqrt{\pi}} \int_{-\infty}^{\infty} \exp \left(\frac{-f^{2}\left(t_{1}-\tau\right)^{2}}{2}\right) \cdot \exp \left(\frac{-f^{2}\left(t_{2}-\tau\right)^{2}}{2}\right) \\
= & \frac{|f|}{\sqrt{\pi}} \int_{-\infty}^{\infty} \exp \left(\frac{-f^{2}\left(t_{1}-\tau\right)^{2}}{2}\right) \cdot \exp \left(\frac{-f^{2}\left(t_{2}-\tau\right)^{2}}{2}\right) \cdot \sin (4 \pi f \tau) d \tau \\
= & \frac{|f|}{j 2 \sqrt{\pi}} \int_{-\infty}^{\infty} \exp \left\{\frac{-f^{2}}{2}\left(2 \tau^{2}-2 \tau\left(t_{1}+t_{2}\right)+\left(t_{1}^{2}+t_{2}^{2}\right)\right)\right\} \\
= & \frac{1}{2 j} \cdot \exp \left(-4 \pi^{2}\right) \cdot \exp \left(\frac{-f^{2}\left(t_{1}-t_{2}\right)^{2}}{4}\right) \cdot \exp \left(j 2 \pi f\left(t_{1}+t_{2}\right)\right) \\
& \cdot \int_{-\infty}^{\infty} \frac{|f|}{\sqrt{\pi}} \cdot \exp \left\{-f^{2}\left(\tau-\left(\frac{t_{1}+t_{2}}{2}+j \frac{2 \pi}{f}\right)\right)^{2}\right\} d \tau \\
& -\frac{1}{2 j} \cdot \exp \left(-4 \pi^{2}\right) \cdot \exp \left(\frac{-f^{2}\left(t_{1}-t_{2}\right)^{2}}{4}\right) \cdot \exp \left(-j 2 \pi f\left(t_{1}+t_{2}\right)\right) \\
& \cdot \int_{-\infty}^{\infty} \frac{|f|}{\sqrt{\pi}} \cdot \exp \left\{-f^{2}\left(\tau-\left(\frac{t_{1}+t_{2}}{2}-j \frac{2 \pi}{f}\right)\right)^{2}\right\} d \tau \\
= & \exp \left(-4 \pi^{2}\right) \cdot \exp \left(\frac{-f^{2}\left(t_{1}-t_{2}\right)^{2}}{4}\right) \cdot \sin \left(2 \pi f\left(t_{1}+t_{2}\right)\right) \approx 0 \\
& \\
& \exp (-j 4 \pi f \tau) d \tau
\end{aligned}
$$


Since both $\operatorname{Re}\left[N S_{n}(t, f)\right]$ and $\operatorname{Im}\left[N S_{n}(t, f)\right]$ follow Gaussian distribution, Equation (10) means they are independent. This concludes the proof that $N S_{n}(t, f)$ has independent and identical distributed real and imaginary parts of standard normal distribution, thus $\left|N S_{n}(t, f)\right|^{2}$ follows $\chi^{2}$ distribution with two degrees of freedom.

\section{Numerical Simulations}

In this section, Monte Carlo methods are used to demonstrate the rationalities of above derivations. The variance of WGN is set to be zero mean and $\sigma^{2}=0.2$ with 512 data points. The results are shown in Table 1, where the theoretical values $\chi_{\alpha}^{2}(n)$ are obtained by $\chi^{2}$ distribution table according to $P\left\{\chi^{2}(n)>\chi_{\alpha}^{2}(n)\right\}=\alpha, n$ is the degree of freedom, $k$ is the times of Monte Carlo methods and the simulation results are obtained by $k$ realizations.

A good agreement between the theoretical values and the simulation results can be observed. And as the times of Monte Carlo experiments increase, the simulation results become closer to their theoretical values. Hence, we have proved our proposition and obtained the distribution of WGN in S transformation domain.

\section{Signal Detection in ST Domain}

In this section, the task of ST based detector is to provide time-varying information in addition to detecting whether a transient signal is present or absent. We use the concept of frame detection which is similar to the common method in voice activity detection (VAD) and no cumulation operator is used. The time slice of S-spectrum is employed as the frame and the decision function is

$$
\left\{\begin{array}{lll}
H_{0}: & x(t)=n(t) & l<\Gamma \\
H_{1}: & x(t)=s(t)+n(t) & l \geq \Gamma
\end{array}\right.
$$

where $x(t)$ is the observed signal, $s(t)$ is the signal to be detected and $n(t)$ is WGN at the time $t$ (also $t$ the frame index). $\Gamma$ is the detection threshold and its value can be calculated as

$$
\Gamma=\frac{1}{2} \frac{\gamma}{|f|} \cdot E\left\{\left|S_{n}(t, f)\right|^{2}\right\}
$$

where the value of $\gamma$ can be determined by $\chi^{2}$ distribution table according to the specific false alarm probability $P_{F A}$ and the value of $E\left\{\left|S_{n}(t, f)\right|^{2}\right\}$ can be estimated under the assumption that the initialization of $x(t)$ is the noise only. The detection statistic can be expressed as

$$
l=\max \left(\left|S_{x}(f)\right|_{t}^{2} /|f|\right)
$$

where $\left|S_{x}(f)\right|_{t}^{2}$ is the time slice of $\left|S_{x}(t, f)\right|^{2}$ at the time $t$. The constant-false-alarm-rate(CFAR) criterion is employed in our detector. The probability of detection $P_{D}$ is used as the performance variable and its value can be numerically expressed as

$$
P_{D}=(1 / k) \sum_{i=1}^{k}\left(N_{\mathrm{H} 1 \mathrm{H} 1} / N_{\mathrm{H} 1}\right)
$$

where $k$ is the time of Monte Carlo realizations, $N_{\mathrm{H} 1}$ is the number of the transient signal frames and $N_{\mathrm{H} 1 \mathrm{H} 1}$ is that of the correct detection frames.

In the analysis, a transient sinusoid signal under WGN environment is used to illustrate the performance of signal detection in ST domain. The simulation signal is of 500 data points with 150 points pure noise at the beginning segment and the end segment, respectively.

Figure 1 shows an example of frame detection at $N_{\mathrm{H} \mid \mathrm{H} 1}$. In this simulation, 4 different frames are selected and it is obvious that the frame at $t=175$ is the signal frame while others are noise ones. In Figure 2, the detector performance is shown in terms of $P_{D}$ versus SNR curves according to three different $P_{F A}$ under CFAR criteria. The results show that the proposed detector can achieve reliable detection at the noise level higher than OdB.

Figure 3 depicts the performance comparison of proposed detector with the same frame detector which uses STFT instead of ST. The threshold corresponding to specific $P_{F A}$ of STFT based detector is obtained by Monte Carlo method of 10000 realizations. The superiority of frame detection is to provide time-varying information. Thus in Figure 4, the initiation time detection result of the transient sinusoid signal is shown under the error tolerance of 1 frame. The simulation result essentially means that this linear time-frequency representation could be potentially used for accurate estimation of the time-varying parameters for nonstationary signals.

\section{Conclusion}

In this paper, the characteristic analysis of white Gaussian

Table 1. Simulation results of $\chi h_{\alpha}^{2}()$ according to $P\left\{\chi^{2}(n)>\chi_{a}^{2}(n)\right\}=$ by $\boldsymbol{k}$ realizations.

\begin{tabular}{cccccc}
\hline$\alpha$ & 0.025 & 0.05 & 0.1 & 0.25 & 0.75 \\
\hline theoretical & 7.378 & 5.991 & 4.605 & 2.773 & 0.575 \\
$k=1000$ & 7.4969 & 6.0005 & 4.6058 & 2.7514 & 0.5640 \\
$k=10000$ & 7.3757 & 5.9915 & 4.6036 & 2.7628 & 0.5732 \\
\hline \multicolumn{1}{c}{$\alpha$} & 0.90 & 0.95 & 0.975 & 0.99 & 0.995 \\
theoretical & 0.211 & 0.103 & 0.051 & 0.020 & 0.010 \\
$k=1000$ & 0.2079 & 0.1006 & 0.0500 & 0.0218 & 0.0108 \\
$k=10000$ & 0.2096 & 0.1025 & 0.0504 & 0.0203 & 0.0102 \\
\hline
\end{tabular}




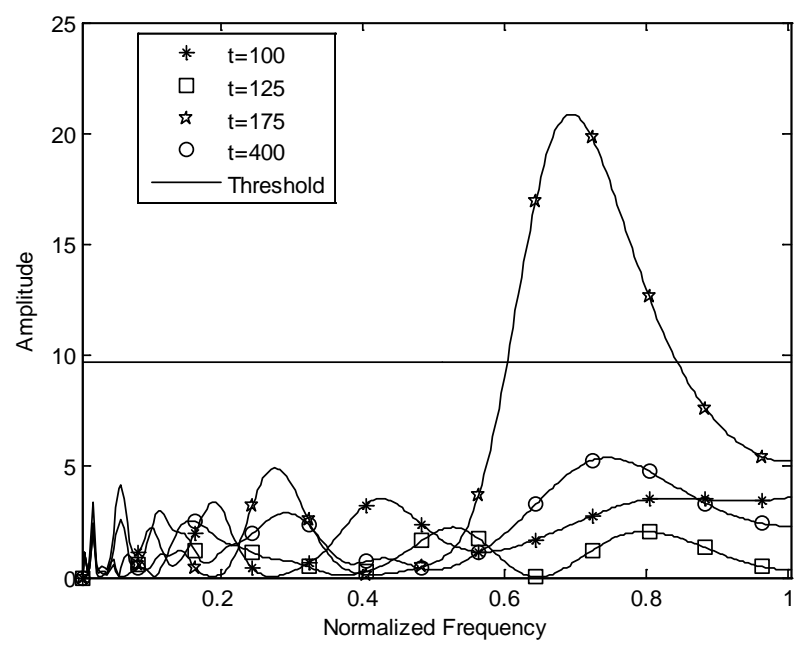

Figure 1. Frame detection results at four different indexes under the noise level of $S N R=0 \mathrm{~dB}$.

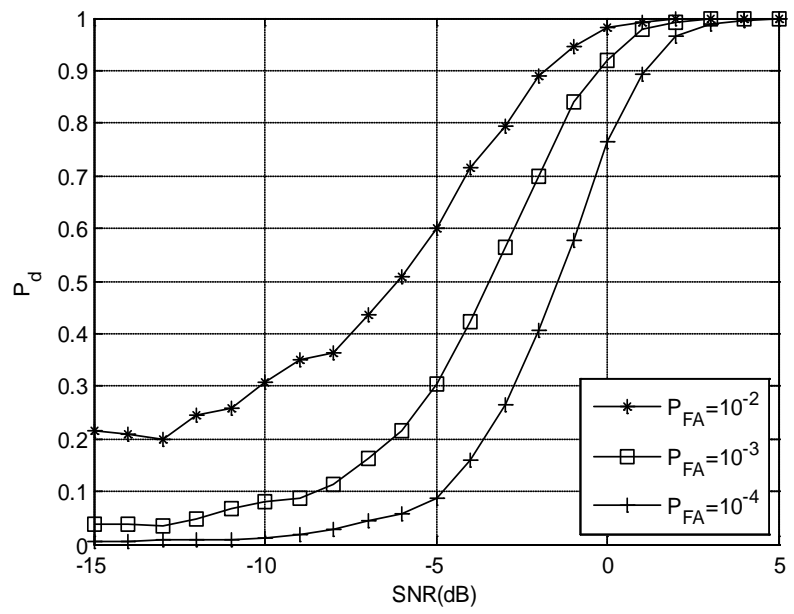

Figure 2. SNR - $P_{D}$ curve for presented detector under three different false alarm probabilities.

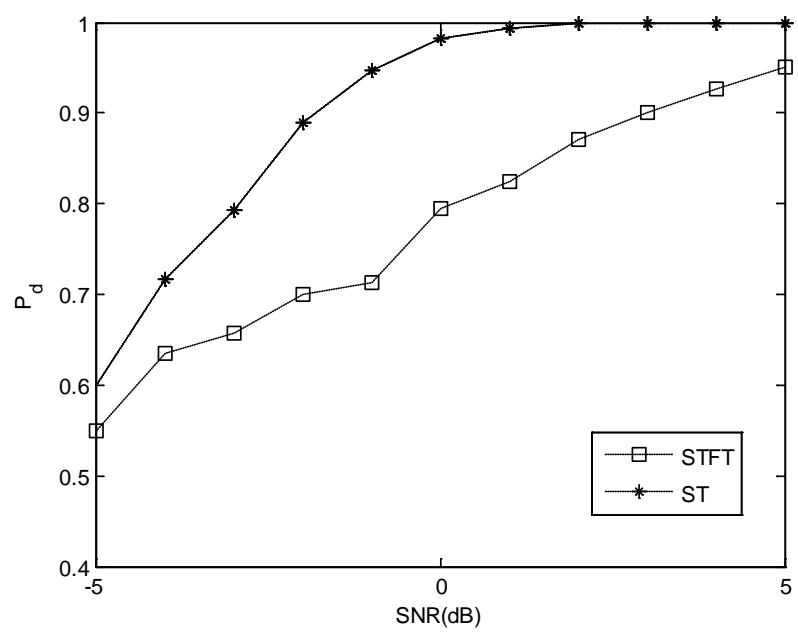

Figure 3. Detection performance of frame detector based on ST and STFT at $P_{F A}=10^{-2}$.

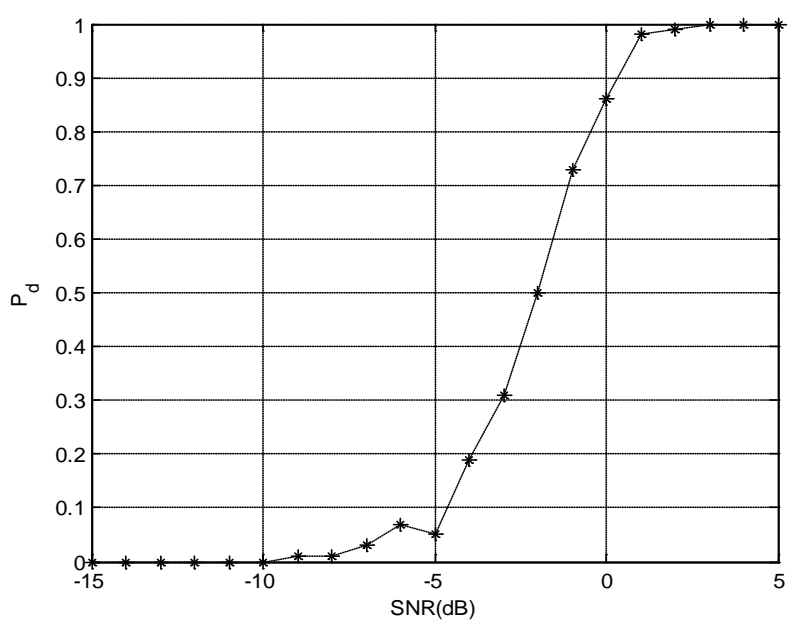

Figure 4. Initiation time detection results of transient sinusoid signals using frame detection.

noise in $\mathrm{S}$ transformation domain has been performed. Accurate distribution of WGN has been analytically derived and been further verified through numerical analysis. This result could be useful in signal detection and ST-based signal processing scheme.

\section{Acknowledgments}

This research was supported in part by a National Natural Science Foundation of China (61301286, 61201287) and a Fundamental Research Funds for the Central Universities (K50511020022).

\section{REFERENCES}

[1] R. G. Stockwell, L. Mansinha and R. P. Lowe, "Localization of the Complex Spectrum: The S-Transform,” IEEE Transactions on Signal Processing, Vol. 44, No. 4, 1996, pp. 998-1001. http://dx.doi.org/10.1109/78.492555

[2] C. R. Pinnegar and L. Mansinha, "The S-Transform with Windows of Arbitrary and Varying Shape,” Geophysics, Vol. 68, No. 1, 2003, pp. 381-385. http://dx.doi.org/10.1190/1.1543223

[3] A. G. Rehorn, E. Sejdic and J. Jiang, "Fault Diagnosis in Machine Tools Using Selective Regional Correlation," Mechanical Systems and Signal Processing, Vol. 20, No. 5, 2006, pp. 1221-1238. http://dx.doi.org/10.1016/j.ymssp.2005.01.010

[4] G. Livanos, N. Ranganathan and J. Jiang, "Heart Sound Analysis Using the S-Transform," Proceedings of Computers in Cardiology, Cambridge, 24-27 September 2000, 587-590.

[5] C. R. Pinnegar and L. Mansinha, "The Bi-Gaussian STransform,” SIAM: SIAM Journal on Scientific Computing, Vol. 24, No. 5, 2003, pp. 1678-1692. http://dx.doi.org/10.1137/S1064827500369803

[6] C.R. Pinnegar, L. Mansinha, “Time-Local Fourier Analysis with a Scalable, Phase-Modulated Analyzing Function: 
The S-Transform with a Complex Window," Signal Process, Vol. 84, No. 7, 2004, pp. 1167-1176. http://dx.doi.org/10.1016/j.sigpro.2004.03.015

[7] M. Schimmel and J. Gallart, "The Inverse S-Transform in Filters with Time-Frequency Localization,” IEEE Transactions on Signal Process, Vol. 53, No. 11, 2005, pp. 4417-4422. http://dx.doi.org/10.1109/TSP.2005.857065

[8] C. Simon, S. Ventosa, M. Schimmel, A. Heldring, J. J. Dañobeitia, J. Gallart and A. Mànuel, "The S-Transform and Its Inverses: Side Effects of Discretizing and Filtering,” IEEE Transactions on Signal Processing, Vol. 55, No. 10, 2007, pp. 4928-4937. http://dx.doi.org/10.1109/TSP.2007.897893
[9] E. Sejdic, L. Stankovic, M. Dakovic and J. Jiang, "Instantaneous Frequency Estimation Using the S-Transform,” IEEE Signal Processing Letters, Vol. 15, 2008, pp. 309-312. http://dx.doi.org/10.1109/LSP.2008.917014

[10] E. Sejdic and J. Jiang, "Selective Regional Correlation for Pattern Recognition," IEEE Transactions on Systems, Man, and Cybernetics, Vol. 37, No. 1, 2007, pp. 82-93.

[11] J. H. Gao, W. S. Man and S. M. Chen, "Recognition of Signals from Colored Noise Background in Generalized S-Transformation Domain," Chinese Journal of Geophysics, Vol. 47, No. 5, 2004, pp. 869-875.

http://dx.doi.org/10.1002/cjg2.576 